\title{
On Quotients of Non-Archimedean Köthe Spaces
}

\author{
Wiesław Śliwa
}

Abstract. We show that there exists a non-archimedean Fréchet-Montel space $W$ with a basis and with a continuous norm such that any non-archimedean Fréchet space of countable type is isomorphic to a quotient of $W$. We also prove that any non-archimedean nuclear Fréchet space is isomorphic to a quotient of some non-archimedean nuclear Fréchet space with a basis and with a continuous norm.

\section{Introduction}

In this paper all linear spaces are over a non-archimedean non-trivially valued field $\mathbb{K}$ which is complete under the metric induced by the valuation $|\cdot|: \mathbb{K} \rightarrow[0, \infty)$. For fundamentals of locally convex Hausdorff spaces (lcs) and normed spaces we refer to $[4,5,6]$.

In $[9,10]$ we investigated closed subspaces in Fréchet spaces of countable type. In this paper we study quotients of Fréchet spaces of countable type.

By a Köthe space we mean a Fréchet space with a basis and with a continuous norm. First, we prove that any Fréchet space of countable type is isomorphic to a quotient of some Köthe space $V$ (Theorem 3 and Corollary 4) and any Köthe space is isomorphic to a quotient of some Köthe-Montel space (Theorem 5). Thus any Fréchet space of countable type is isomorphic to a quotient of some Köthe-Montel space $W$ (Corollary 6).

Next, we show that any nuclear Fréchet space is isomorphic to a quotient of some nuclear Köthe space Theorem 7, but there is no nuclear Fréchet space $X$ such that any nuclear Köthe space is isomorphic to a quotient of $X$ (Theorem 10 and Corollary 12).

\section{Preliminaries}

The linear span of a subset $A$ of a linear space $E$ is denoted by lin $A$.

Let $E, F$ be locally convex spaces. A map $T: E \rightarrow F$ is called an isomorphism if $T$ is linear, injective, surjective and the maps $T, T^{-1}$ are continuous. $E$ is isomorphic to $F$ if there exists an isomorphism $T: E \rightarrow F$.

A seminorm on a linear space $E$ is a function $p: E \rightarrow[0, \infty)$ such that $p(\alpha x)=$ $|\alpha| p(x)$ for all $\alpha \in \mathbb{K}, x \in E$ and $p(x+y) \leq \max \{p(x), p(y)\}$ for all $x, y \in E$. A seminorm $p$ on $E$ is a norm if $\operatorname{ker} p=\{0\}$.

Received by the editors 2004-04-29.

AMS subject classification: 46S10, 46A45.

Keywords: Non-archimedean Köthe spaces, nuclear Fréchet spaces, pseudo-bases.

(c) Canadian Mathematical Society ZZZZ. 
The set of all continuous seminorms on a metrizable lcs $E$ is denoted by $\mathcal{P}(E)$. A non-decreasing sequence $\left(p_{k}\right) \subset \mathcal{P}(E)$ is a base in $\mathcal{P}(E)$ if for every $p \in \mathcal{P}(E)$ there exists $k \in \mathbb{N}$ with $p \leq p_{k}$. A sequence $\left(p_{k}\right)$ of norms on $E$ is a base of norms in $\mathcal{P}(E)$ if it is a base in $\mathcal{P}(E)$.

Any metrizable lcs $E$ possesses a base $\left(p_{k}\right)$ in $\mathcal{P}(E)$.

A metrizable lcs $E$ is of finite type if $\operatorname{dim}(E / \operatorname{ker} p)<\infty$ for any $p \in \mathcal{P}(E)$, and of countable type if $E$ contains a linearly dense countable set.

A Fréchet space is a metrizable complete lcs. Any infinite-dimensional Fréchet space of finite type is isomorphic to the Fréchet space $\mathbb{K}^{\mathbb{N}}$ of all sequences in $\mathbb{K}$ with the topology of pointwise convergence (see [2, Theorem 3.5]).

Let $\left(x_{n}\right)$ be a sequence in a Fréchet space $E$. The series $\sum_{n=1}^{\infty} x_{n}$ is convergent in $E$ if and only if $\lim x_{n}=0$.

A sequence $\left(x_{n}\right)$ in an lcs $E$ is a basis in $E$ if each $x \in E$ can be written uniquely as $x=\sum_{n=1}^{\infty} \alpha_{n} x_{n}$ with $\left(\alpha_{n}\right) \subset \mathbb{K}$. If additionally the coefficient functionals $f_{n}: E \rightarrow$ $\mathbb{K}, x \rightarrow \alpha_{n},(n \in \mathbb{N})$ are continuous, then $\left(x_{n}\right)$ is a Schauder basis in E. As in the real and complex case any basis in a Fréchet space is a Schauder basis (see [3, Corollary 4.2]).

A Banach space is a normable Fréchet space. Any infinite-dimensional Banach space $E$ of countable type is isomorphic to the Banach space $c_{0}$ of all sequences in $\mathbb{K}$ converging to zero with the sup-norm [5, Theorem 3.16].

Let $p$ be a seminorm on a linear space $E$ and $t \in(0,1)$. A sequence $\left(x_{n}\right)$ in $E$ is $t$-orthogonal with respect to $p$ if $p\left(\sum_{i=1}^{n} \alpha_{i} x_{i}\right) \geq t \max _{1 \leq i \leq n} p\left(\alpha_{i} x_{i}\right)$ for all $n \in$ $\mathbb{N}, \alpha_{1}, \ldots, \alpha_{n} \in \mathbb{K}$.

A sequence $\left(x_{n}\right)$ in an lcs $E$ is 1-orthogonal with respect to $\left(p_{k}\right) \subset \mathcal{P}(E)$ provided $p_{k}\left(\sum_{i=1}^{n} \alpha_{i} x_{i}\right)=\max _{1 \leq i \leq n} p_{k}\left(\alpha_{i} x_{i}\right)$ for all $k, n \in \mathbb{N}, \alpha_{1}, \ldots, \alpha_{n} \in \mathbb{K}$.

Every basis $\left(x_{n}\right)$ in a Fréchet space $E$ is 1-orthogonal with respect to some basis $\left(p_{k}\right)$ in $\mathcal{P}(E)$ [2, Proposition 1.7].

Let $B=\left(b_{n, k}\right)$ be an infinite real matrix with $0<b_{n, k} \leq b_{n, k+1} \forall n, k \in \mathbb{N}$. The space $K(B)=\left\{\left(\alpha_{n}\right) \subset \mathbb{K}: \lim _{n}\left|\alpha_{n}\right| b_{n, k}=0\right.$ for all $\left.k \in \mathbb{N}\right\}$ with the base of norms $\left(p_{k}\right): p_{k}\left(\left(\alpha_{n}\right)\right)=k \max _{n}\left|\alpha_{n}\right| b_{n, k}, k \in \mathbb{N}$, is a Köthe space. The sequence $\left(e_{n}\right)$ of coordinate vectors forms a basis in $K(B)$; the coordinate basis is 1-orthogonal with respect to the base $\left(p_{k}\right)$ [1, Proposition 2.2].

Put $B_{\mathbb{K}}=\{\alpha \in \mathbb{K}:|\alpha| \leq 1\}$. Let $A$ be a subset of an lcs $E$. The set $\operatorname{co} A=$ $\left\{\sum_{i=1}^{n} \alpha_{i} a_{i}: n \in \mathbb{N}, \alpha_{1}, \ldots, \alpha_{n} \in B_{\mathbb{K}}, a_{1}, \ldots, a_{n} \in A\right\}$ is the absolutely convex hull of $A$; its closure in $E$ is denoted by $\overline{\operatorname{co}} A$.

A subset $B$ of an lcs $E$ is absolutely convex if $\operatorname{co} B=B$.

A subset $B$ of an lcs $E$ is compactoid if for each neighbourhood $U$ of 0 in $E$ there exists a finite subset $A$ of $E$ such that $B \subset U+\operatorname{co} A$.

By a Fréchet-Montel space we mean a Fréchet space in which any bounded subset is compactoid.

Let $E$ and $F$ be locally convex spaces. A linear map $T: E \rightarrow F$ is compact if there exists a neighbourhood $U$ of 0 in $E$ such that $T(U)$ is compactoid in $F$.

For any seminorm $p$ on an lcs $E$ the map $\bar{p}: E_{p} \rightarrow[0, \infty), x+\operatorname{ker} p \rightarrow p(x)$ is a norm on $E_{p}=(E / \operatorname{ker} p)$. Let $\varphi_{p}: E \rightarrow E_{p}, x \rightarrow x+\operatorname{ker} p$.

An lcs $E$ is nuclear if for every continuous seminorm $p$ on $E$ there exists a contin- 
uous seminorm $q$ on $E$ with $q \geq p$ such that the map

$$
\varphi_{p q}:\left(E_{q}, \bar{q}\right) \rightarrow\left(E_{p}, \bar{p}\right), x+\operatorname{ker} q \rightarrow x+\operatorname{ker} p
$$

is compact.

Let $E$ be a Fréchet space with a basis $\left(x_{n}\right)$ which is 1-orthogonal with respect to a base of norms $\left(p_{k}\right)$ in $\mathcal{P}(E)$. Then $E$ is nuclear if and only if $\forall k \in \mathbb{N}, \exists m>k$ : $\lim _{n}\left[p_{k}\left(x_{n}\right) / p_{m}\left(x_{n}\right)\right]=0$ [1, Propositions 2.4 and 3.5].

\section{Results}

A sequence $\left(x_{n}\right)$ in a Fréchet space $X$ is a pseudo-basis of $X$, if for any element $x$ of $X$ there is a sequence $\left(\alpha_{n}\right) \subset \mathbb{K}$ such that the series $\sum_{n=1}^{\infty} \alpha_{n} x_{n}$ is convergent in $X$ to $x$.

In [8] we have proved that there exist nuclear Fréchet spaces without a basis. For pseudo-bases we have the following.

\section{Proposition 1 Any Fréchet space E of countable type has a pseudo-basis.}

Proof Let $\left(p_{k}\right)$ be a base in $\mathcal{P}(E)$ and $U_{k}=\left\{x \in E: p_{k}(x) \leq 1\right\}, k \in \mathbb{N}$. Let $\beta \in \mathbb{K}$ with $0<|\beta|<1$. Choose a linearly independent and linearly dense sequence $\left(z_{i}\right)$ in $E$. Put $Z_{n}=\operatorname{lin}\left\{z_{i}: 1 \leq i \leq n\right\}, n \in \mathbb{N}$. Let $\left(N_{k}\right)$ be a partition of $\mathbb{N}$ into infinite subsets. For $n \in N_{k}, k \in \mathbb{N}$, let $x_{n, 1}, \ldots, x_{n, n}$ be a basis in $Z_{n}$ which is $|\beta|$-orthogonal with respect to $p_{k}$ (see [10, proof of Lemma 1.1]). We will show that the sequence $\left(x_{n}\right)=\left(x_{1,1}, x_{2,1}, x_{2,2}, x_{3,1}, x_{3,2}, x_{3,3}, \ldots\right)$ is a pseudo-basis in $E$.

Let $k \in \mathbb{N}, x \in U_{k}$ and $m \in \mathbb{N}$. Then for some $n \in N_{k}$ with $n \geq m$ there is $y \in Z_{n} \cap\left(x+U_{k+1}\right)$. Thus $\exists \beta_{1}, \ldots, \beta_{n} \in \mathbb{K}: y=\sum_{i=1}^{n} \beta_{i} x_{n, i}$ and

$$
|\beta| \max _{1 \leq i \leq n} p_{k}\left(\beta_{i} x_{n, i}\right) \leq p_{k}(y) \leq \max \left\{p_{k}(y-x), p_{k}(x)\right\} \leq 1 .
$$

Hence $\beta_{1} x_{n, 1}, \ldots, \beta_{n} x_{n, n} \in \beta^{-1} U_{k}$.

We have proved that $\forall k \in \mathbb{N}, \forall x \in U_{k}, \forall m \in \mathbb{N}, \exists s \geq m, \exists \alpha_{m}, \ldots, \alpha_{s} \in \mathbb{K}$ :

$$
\left(x-\sum_{i=m}^{s} \alpha_{i} x_{i}\right) \in U_{k+1} \text { and }\left\{\alpha_{m} x_{m}, \ldots, \alpha_{s} x_{s}\right\} \subset \beta^{-1} U_{k} .
$$

It follows that the sequence $\left(x_{n}\right)$ is a pseudo-basis in $E$.

Remark 2 It is easy to see that any dense sequence $\left(x_{n}\right)$ in a Fréchet space $E$ is a pseudo-basis of $E$. Unfortunately, any non-zero Fréchet space over a non-separable field is non-separable.

Using the existence of pseudo-bases in any Fréchet space of countable type we get the following.

Theorem 3 Any Fréchet space E of countable type is isomorphic to a quotient of some Köthe space. 
Proof Assume that $E$ is not of finite type. Then for some $p \in \mathcal{P}(E)$ the quotient space $(E / \operatorname{ker} p)$ is infinite-dimensional. Let $G$ be an algebraic complement of $\operatorname{ker} p$ in $E$. Since $G$ is an infinite-dimensional metrizable lcs of countable type, it contains a linearly independent and linearly dense sequence $\left(g_{n}\right)$. Let $\left(s_{k}\right)$ be a linearly dense sequence in $\operatorname{ker} p$ and let $\left(N_{k}\right)$ be a partition of $\mathbb{N}$ into infinite subsets. We can choose a sequence $\left(\alpha_{n}\right) \subset(\mathbb{K} \backslash\{0\})$ with $\lim _{n \in N_{k}} \alpha_{n} g_{n}=0, k \in \mathbb{N}$. Put $z_{n}=\alpha_{n} g_{n}+s_{k}$ for $n \in N_{k}, k \in \mathbb{N}$. The sequence $\left(z_{n}\right)$ is linearly independent and linearly dense in $E$, and $\operatorname{lin}\left(z_{n}\right) \cap \operatorname{ker} p=\{0\}$.

By Proposition 1 and its proof, the space $E$ has a pseudo-basis $\left(e_{n}\right)$ such that $\left(e_{n}\right) \subset$ $\left(\operatorname{lin}\left(z_{n}\right) \backslash\{0\}\right)$. Let $\left(p_{k}\right)$ be a base in $\mathcal{P}(E)$ with $p_{1} \geq p$. Put $a_{n, k}=p_{k}\left(e_{n}\right)$ for $n, k \in \mathbb{N}$. Clearly, $0<a_{n, k} \leq a_{n, k+1}$ for all $n, k \in \mathbb{N}$. Let $A=\left(a_{n, k}\right)$ and let $X$ be the Köthe space $K(A)$.

For any $\alpha=\left(\alpha_{n}\right) \in X$ the series $\sum_{n=1}^{\infty} \alpha_{n} e_{n}$ is convergent in $E$. Moreover, $p_{k}\left(\sum_{n=1}^{\infty} \alpha_{n} e_{n}\right) \leq \max _{n}\left|\alpha_{n}\right| a_{n, k} \leq q_{k}(\alpha)$ for $k \in \mathbb{N}, \alpha \in X$, where $\left(q_{k}\right)$ is the standard base of norms in $\mathcal{P}(X)$. Thus the linear operator $T: X \rightarrow E, T \alpha=\sum_{n=1}^{\infty} \alpha_{n} e_{n}$, is well defined and continuous. We show that $T(X)=E$. Let $e \in E$. Then there exists $\left(\alpha_{n}\right) \subset \mathbb{K}$ such that $\sum_{n=1}^{\infty} \alpha_{n} e_{n}=e$. Clearly, $\lim _{n}\left|\alpha_{n}\right| a_{n, k}=\lim _{n}\left|\alpha_{n}\right| p_{k}\left(x_{n}\right)=0$, $k \in \mathbb{N}$. Thus $\alpha=\left(\alpha_{n}\right) \in X$ and $T \alpha=e$. It follows that $E$ is isomorphic to the quotient $(X / \operatorname{ker} T)$ of $X$.

If $E$ is of finite type, then it is isomorphic to a quotient of $\mathbb{K}^{\mathbb{N}} \times c_{0}$ and, by the first part of the proof, to a quotient of some Köthe space.

In [12] we have proved that there exists a Köthe space $V$ (unique up to isomorphism) such that any Köthe space is isomorphic to a complemented closed subspace of $V$. Thus, by Theorem 3, we get

Corollary 4 Any Fréchet space of countable type is isomorphic to a quotient of the Köthe space $V$.

Now we prove the following.

Theorem 5 Any Köthe space $X$ is isomorphic to a quotient of some Köthe-Montel space.

Proof Let $\left(x_{n}\right)$ be a basis in $X$. This basis is 1-orthogonal with respect to a base of norms $\left(p_{k}\right)$ in $\mathcal{P}(X)$. Without loss of generality we can assume that $p_{1}\left(x_{n}\right) \geq 1, n \in$ $\mathbb{N}$. Put $d_{m, k}=p_{k}\left(x_{m}\right)$ for $m, k \in \mathbb{N}$. Let $\left(N_{i}\right),\left(S_{m}\right)$ be two partitions of $\mathbb{N}$ such that the set $N_{i} \cap S_{m}$ is non-empty for all $i, m \in \mathbb{N}$.

For $n \in N_{i} \cap S_{m}, i, m \in \mathbb{N}$ and $k \in \mathbb{N}$ we put $b_{n, k}=k^{i} d_{m, k}$ if $k \leq i$ and $b_{n, k}=$ $k^{\text {in }} d_{m, k}$ if $k>i$. Clearly, $0<b_{n, k} \leq b_{n, k+1}$ for all $n, k \in \mathbb{N}$. Put $B=\left(b_{n, k}\right)$. The Köthe space $K(B)$ is a Fréchet-Montel space (see [10, Corollary 1.10, Example 1.9 and its proof]). We will prove that $X$ is isomorphic to a quotient of $K(B)$. Put $Y=K(B)$.

Let $\left(f_{n}\right) \subset Y^{\prime}$ be the sequence of coefficient functionals associated with the coordinate basis $\left(e_{n}\right)$ in $Y$. For any $\alpha=\left(\alpha_{n}\right) \in Y$ we have $\lim _{n} f_{n}(\alpha)=0$, since 
$\lim _{n}\left|\alpha_{n}\right| b_{n, 1}=0$. Put $g_{m}(\alpha)=\sum_{n \in S_{m}} f_{n}(\alpha)$ for $m \in \mathbb{N}$ and $\alpha \in Y$. By the BanachSteinhaus theorem, the linear functionals $g_{m}, m \in \mathbb{N}$, are continuous on $Y$. For all $k, m \in \mathbb{N}$ and $\alpha \in Y$ we have

$$
p_{k}\left(g_{m}(\alpha) x_{m}\right)=\left|g_{m}(\alpha)\right| d_{m, k} \leq \sup _{n \in S_{m}}\left|f_{n}(\alpha)\right| d_{m, k} \leq \sup _{n \in S_{m}}\left|\alpha_{n}\right| b_{n, k}
$$

and $\lim _{n}\left|\alpha_{n}\right| b_{n, k}=0$, so $\lim _{m} g_{m}(\alpha) x_{m}=0$ in $X$, for any $\alpha \in Y$. Put $T: Y \rightarrow$ $X, T \alpha=\sum_{m=1}^{\infty} g_{m}(\alpha) x_{m}$. For $k, m \in \mathbb{N}$ and $\alpha \in Y$ we get

$$
p_{k}(T \alpha) \leq \max _{m} \max _{n \in S_{m}}\left|f_{n}(\alpha)\right| d_{m, k} \leq \max _{m} \max _{n \in S_{m}} q_{k}(\alpha)\left(d_{m, k} b_{n, k}^{-1}\right) \leq q_{k}(\alpha),
$$

where $\left(q_{k}\right)$ is the standard base of norms in $\mathcal{P}(Y)$. Thus the linear operator $T$ is continuous. We show that $T(Y)=X$. Let $x \in X$. Then $\exists\left(\alpha_{m}\right) \subset \mathbb{K}: x=\sum_{m=1}^{\infty} \alpha_{m} x_{m}$ and $\forall k \in \mathbb{N}, \lim _{m}\left|\alpha_{m}\right| d_{m, k}=0$. Therefore there exists an increasing sequence $\left(m_{k}\right) \subset \mathbb{N}$ with $m_{1}=1$ such that $\left|\alpha_{m}\right| d_{m, k} \leq k^{-k-1} p_{1}(x)$ for $m_{k} \leq m<m_{k+1}, k \in \mathbb{N}$. Let $t_{m} \in N_{k} \cap S_{m}$ for $m_{k} \leq m<m_{k+1}, k \in \mathbb{N}$. Let $l \in \mathbb{N}$. Then for $k \geq l$ and $m_{k} \leq m<m_{k+1}$ we have

$$
\left|\alpha_{m}\right| b_{t_{m}, l} \leq\left|\alpha_{m}\right| b_{t_{m}, k}=\left|\alpha_{m}\right| d_{m, k} k^{k} \leq k^{-1} p_{1}(x) .
$$

Hence $\forall l \in \mathbb{N}, \lim _{m}\left|\alpha_{m}\right| b_{t_{m}, l}=0$. Thus the series $\sum_{m=1}^{\infty} \alpha_{m} e_{t_{m}}$ is convergent in $Y$ to some element $y$. Clearly, $T y=x$; so $T(Y)=X$. It follows that $X$ is isomorphic to the quotient $(Y / \operatorname{ker} T)$ of $Y$.

By Corollary 4 and Theorem 5 we obtain

Corollary 6 Any Fréchet space of countable type is isomorphic to a quotient of some Köthe-Montel space W.

For nuclear Fréchet spaces we shall prove the following.

Theorem 7 Any nuclear Fréchet space E is isomorphic to a quotient of some nuclear Köthe space.

Proof Assume that $E$ is not of finite type. Let $\beta \in \mathbb{K}$ with $0<|\beta|<1$. Then $E$ possesses a base $\left(p_{k}\right)$ in $\mathcal{P}(E)$ such that:

(1) $\operatorname{dim}\left(E / \operatorname{ker} p_{1}\right)=\infty$;

(2) $\forall k \in \mathbb{N}, p_{k} \leq|\beta|^{2} p_{k+1}$;

(3) for any $k \in \mathbb{N}$ the canonical map $\varphi_{k, k+1}:\left(E_{k+1}, \overline{p_{k+1}}\right) \rightarrow\left(E_{k}, \overline{p_{k}}\right)$ is compact.

Let $\left(z_{n}\right)$ be a linearly independent and linearly dense sequence in $E$ such that $\operatorname{lin}\left(z_{n}\right) \cap \operatorname{ker} p_{1}=\{0\}$ (see the proof of Theorem 3). Put $Z=\operatorname{lin}\left(z_{n}\right)$ and $U_{m}=$ $\left\{x \in E: p_{m}(x) \leq 1\right\}$ for $m \in \mathbb{N}$. Let $k \in \mathbb{N}$.

Let $\left(v_{n}\right)$ be a $|\beta|$-orthogonal basis in $\left(E_{k+1}, \overline{p_{k+1}}\right)$ with $|\beta|<\overline{p_{k+1}}\left(v_{n}\right) \leq 1, n \in \mathbb{N}$, such that $\operatorname{lin}\left(v_{n}\right)=\operatorname{lin}\left(\varphi_{k+1}\left(z_{n}\right)\right)$ (see [5], Theorem 3.16 (i) and its proof). Put $u_{n}=\left(\varphi_{k+1} \mid Z\right)^{-1}\left(v_{n}\right), n \in \mathbb{N}$. Then $\left(u_{n}\right) \subset Z \cap U_{k+1}$. 
We will show that $U_{k+2} \subset \overline{\mathrm{co}}\left(u_{n}\right)$. Let $x \in U_{k+2}$. Assume $m \in \mathbb{N}, \alpha_{1}, \ldots, \alpha_{m} \in \mathbb{K}$ and $\left(x-\sum_{i=1}^{m} \alpha_{i} u_{i}\right) \in U_{k+2}$. Then

$$
p_{k+1}\left(\sum_{i=1}^{m} \alpha_{i} u_{i}\right) \leq \max \left\{p_{k+1}\left(\sum_{i=1}^{m} \alpha_{i} u_{i}-x\right), p_{k+1}(x)\right\} \leq|\beta|^{2}
$$

and

$$
p_{k+1}\left(\sum_{i=1}^{m} \alpha_{i} u_{i}\right)=\overline{p_{k+1}}\left(\sum_{i=1}^{m} \alpha_{i} v_{i}\right) \geq|\beta| \max _{1 \leq i \leq m} \overline{p_{k+1}}\left(\alpha_{i} v_{i}\right) \geq|\beta|^{2} \max _{1 \leq i \leq m}\left|\alpha_{i}\right| .
$$

Hence $\max _{1 \leq i \leq m}\left|\alpha_{i}\right| \leq 1$. We have proved that $\sum_{i=1}^{m} \alpha_{i} u_{i} \in \operatorname{co}\left(u_{n}\right)$ provided $\left(x-\sum_{i=1}^{m} \alpha_{i} u_{i}\right) \in U_{k+2}$. Thus $x \in \overline{\mathrm{co}}\left(u_{n}\right)$, since $\left(u_{n}\right)$ is linearly dense in $E$. Hence $U_{k+2} \subset \overline{\operatorname{co}}\left(u_{n}\right)$.

Put $W=Z \cap U_{k+1}$. The set $\varphi_{k}(W)$ is absolutely convex and compactoid in $\left(E_{k}, \overline{p_{k}}\right)$. Therefore there exists a sequence $\left(y_{i}\right) \subset\left(\beta^{-1} \varphi_{k}(W) \backslash\{0\}\right)$ with $\lim _{i} \overline{p_{k}}\left(y_{i}\right)=0$ such that $\varphi_{k}(W) \subset \overline{\mathrm{co}}\left(y_{i}\right)$ (see [6, Proposition 8.2]).

Let $d_{i} \in \beta^{-1} W$ with $\varphi_{k}\left(d_{i}\right)=y_{i}, i \in \mathbb{N}$. Clearly, $0<p_{k}\left(d_{i}\right) \leq|\beta|, i \in \mathbb{N}$, and $\lim _{i} p_{k}\left(d_{i}\right)=0$. Since $\left(u_{n}\right) \subset Z \cap U_{k+1}$, we have

$$
\forall n \in \mathbb{N}, \exists m \in \mathbb{N}, \exists \alpha_{1}, \ldots, \alpha_{m} \in B_{\mathbb{K}}: 0<\overline{p_{k}}\left(\varphi_{k}\left(u_{n}\right)-\sum_{i=1}^{m} \alpha_{i} y_{i}\right)<n^{-1} .
$$

Put $b_{n}=u_{n}-\sum_{i=1}^{m} \alpha_{i} d_{i}, n \in \mathbb{N}$. Then $0<p_{k}\left(b_{n}\right)<n^{-1}, n \in \mathbb{N}$.

Let $x_{2 n-1}^{k}=d_{n}, x_{2 n}^{k}=b_{n}$ for $n \in \mathbb{N}$. Clearly, $\left(x_{n}^{k}\right) \subset Z \cap\left(U_{k} \backslash\{0\}\right), \lim _{n} p_{k}\left(x_{n}^{k}\right)=0$ and $\left(u_{n}\right) \subset \operatorname{co}\left(x_{n}^{k}\right)$; hence $U_{k+2} \subset \overline{\mathrm{co}}\left(u_{n}\right) \subset \overline{\mathrm{co}}\left(x_{n}^{k}\right)$.

Let $\left(S_{k}\right)$ be a partition of $\mathbb{N}$ into infinite subsets and let $\left(x_{n}\right)$ be a sequence in $E$ such that $\left(x_{n}\right)_{n \in S_{k}}=\left(x_{1}^{k}, x_{2}^{k}, \ldots\right)$ for any $k \in \mathbb{N}$. Let $d_{n, k}=p_{k}\left(x_{n}\right)$ for $n, k \in \mathbb{N}$. Clearly, $0<d_{n, k} \leq d_{n, k+1}$ for $n, k \in \mathbb{N}$. Moreover, $0<d_{n, m} \leq 1$ for $n \in S_{m}, m \in \mathbb{N}$, and $\lim _{n \in S_{m}} d_{n, m}=0, m \in \mathbb{N}$.

Put $b_{n, k}=d_{n, k} d_{n, m}^{-k / m}|\beta|^{-k m}$ for $n \in S_{m}, m \in \mathbb{N}$, and $k \in \mathbb{N}$. Clearly, $0<b_{n, k} \leq$ $|\beta| b_{n, k+1}$ for all $n, k \in \mathbb{N}$. Let $k \in \mathbb{N}$. For $n \in S_{m}, m \in \mathbb{N}$, we have $b_{n, k} b_{n, k+1}^{-1} \leq$ $d_{n, m}^{1 / m}|\beta|^{m}$. Let $\epsilon>0$. Then $\exists l \in \mathbb{N}, \forall m>l,|\beta|^{m} \leq \epsilon$ and $\exists t \in \mathbb{N}, \forall 1 \leq m \leq l, \forall n \in$ $\left(S_{m} \backslash\{1, \ldots, t\}\right), d_{n, m} \leq \epsilon^{m}$. Hence $\forall n>t, b_{n, k} b_{n, k+1}^{-1} \leq \epsilon$. Thus $\lim _{n} b_{n, k} b_{n, k+1}^{-1}=$ $0, k \in \mathbb{N}$; so the Köthe space $K(B)$, associated with the matrix $B=\left(b_{n, k}\right)$, is nuclear.

We shall show that $E$ is isomorphic to a quotient of $K(B)$. Put $Y=K(B)$ and $q_{k}(\alpha)=\max _{n}\left|\alpha_{n}\right| b_{n, k}$ for $\alpha=\left(\alpha_{n}\right) \in Y$ and $k \in \mathbb{N}$. Clearly, $\left(q_{k}\right)$ is a base in $\mathcal{P}(Y)$. Let $\alpha=\left(\alpha_{n}\right) \in Y$ and $k \in \mathbb{N}$. For $n \in S_{m}, m \in \mathbb{N}$ we have

$$
p_{k}\left(\alpha_{n} x_{n}\right)=\left|\alpha_{n}\right| d_{n, k} \leq q_{k}(\alpha) b_{n, k}^{-1} d_{n, k}=q_{k}(\alpha)\left(d_{n, m}^{1 / m}|\beta|^{m}\right)^{k} .
$$

Thus $\lim _{n} p_{k}\left(\alpha_{n} x_{n}\right)=0$ and $\max _{n} p_{k}\left(\alpha_{n} x_{n}\right) \leq q_{k}(\alpha)$ for all $\alpha=\left(\alpha_{n}\right) \in Y$ and $k \in \mathbb{N}$. It follows that the linear map

$$
T: Y \rightarrow E, T \alpha=\sum_{n=1}^{\infty} \alpha_{n} x_{n}
$$


is well defined and continuous. Put $V_{m}=\left\{\alpha \in Y: q_{m}(\alpha) \leq 1\right\}, m \in \mathbb{N}$. Let $\left(e_{n}\right)$ be the coordinate basis in $Y$. Let $m \in \mathbb{N}$. Since $q_{m}\left(\beta^{m^{2}} e_{n}\right)=|\beta|^{m^{2}} b_{n, m}=1$ for $n \in S_{m}$, we have $T\left(V_{m}\right) \supset\left\{\beta^{m^{2}} x_{n}: n \in S_{m}\right\}$; so $\overline{T\left(V_{m}\right)} \supset \beta^{m^{2}} \overline{\operatorname{co}}\left\{x_{n}^{m}: n \in \mathbb{N}\right\} \supset \beta^{m^{2}} U_{m+2}$. Thus the map $T$ is almost open. By the open mapping theorem [4, Theorem 2.72] we infer that $T(Y)=E$ and $E$ is isomorphic to the quotient $(Y / \operatorname{ker} T)$ of $Y$.

If $E$ is of finite type and $K(B)$ is a nuclear Köthe space, then $E$ is isomorphic to a quotient of $\mathbb{K}^{\mathbb{N}} \times K(B)$ and, by the first part of the proof, to a quotient of some nuclear Köthe space.

Finally, we shall show that there is no nuclear Fréchet space $X$ such that any nuclear Köthe space is isomorphic to a quotient of $X$.

For arbitrary subsets $A, B$ in a linear space $E$ and a linear subspace $L$ of $E$ we denote $d(A, B, L)=\inf \{|\beta|: \beta \in \mathbb{K}$ and $A \subset \beta B+L\}$ (we put inf $\varnothing=\infty$ ). Let $d_{n}(A, B)=$ $\inf \{d(A, B, L): L<E$ and $\operatorname{dim} L<n\}, n \in \mathbb{N}$.

It is easy to check the following.

Remark 8 Let $E$ and $F$ be linear spaces. If $A, B \subset E$ and $T$ is a linear map from $E$ onto $F$, then $d_{n}(A, B) \geq d_{n}(T(A), T(B))$ for $n \in \mathbb{N}$. If $A^{\prime} \subset A \subset E$ and $B \subset B^{\prime} \subset E$, then $d_{n}(A, B) \geq d_{n}\left(A^{\prime}, B^{\prime}\right)$ for $n \in \mathbb{N}$.

By the second part of the proof of [11, Lemma 2], we get

Lemma 9 Let $\left(f_{n}\right)$ be the sequence of coefficient functionals associated with a basis $\left(x_{n}\right)$ in an lcs E. Let $\left(a_{k}\right),\left(b_{k}\right) \subset(0, \infty)$. Put $A=\left\{x \in E: \max _{k}\left|f_{k}(x)\right| a_{k}^{-1} \leq 1\right\}$ and $B=\left\{x \in E: \max _{k}\left|f_{k}(x)\right| b_{k}^{-1} \leq 1\right\}$. Then for any $n \in \mathbb{N}$ and $\alpha \in \mathbb{K}$ with $|\alpha|<1$ we have $d_{n}(A, B) \geq|\alpha| a_{n} b_{n}^{-1}$.

If $a=\left(a_{n}\right) \subset(0, \infty)$ is a non-decreasing sequence with $\lim a_{n}=\infty$, then the following Köthe space is nuclear: $A_{\infty}(a)=K(B)$ with $B=\left(b_{k, n}\right), b_{k, n}=k^{a_{n}}$ (see $[1]) ; A_{\infty}(a)$ is a power series space of infinite type.

Now we can prove our last theorem.

Theorem 10 For any nuclear Köthe space $X$ there exists a non-decreasing sequence $\left(a_{n}\right) \subset(0, \infty)$ with $\lim _{n} a_{n}=\infty$ such the space $A_{\infty}(a)$ is not isomorphic to any quotient of $X$.

Proof Let $\beta \in \mathbb{K}$ with $0<|\beta|<1$. Let $\left(x_{n}\right)$ be a basis of $X$ which is 1-orthogonal with respect to a base of norms $\left(p_{k}\right)$ in $\mathcal{P}(X)$ with $\lim _{n}\left[p_{k}\left(x_{n}\right) p_{k+1}^{-1}\left(x_{n}\right)\right]=0, k \in \mathbb{N}$. Put $U_{k}=\left\{x \in X: p_{k}(x) \leq 1\right\}$ for $k \in \mathbb{N}$. It is easy to see that

$$
\forall i \in \mathbb{N}, \forall m \in \mathbb{N}, \exists n \in \mathbb{N}: U_{i+1} \subset \beta^{m} U_{i}+\operatorname{lin}\left\{x_{1}, \ldots, x_{n}\right\} .
$$

Hence $\lim _{n} d_{n}\left(U_{i+1}, U_{i}\right)=0, i \in \mathbb{N}$. Thus there exists an increasing sequence $\left(v_{n}\right) \subset \mathbb{N}$ such that for any $n \in \mathbb{N}$ we have

$$
\max _{1 \leq k \leq n} d_{v_{n}}\left(U_{k+1}, U_{k}\right)<|\beta| n^{-n} .
$$


Put $a_{m}=\min \left\{n \in \mathbb{N}: v_{n} \geq m\right\}, m \in \mathbb{N}$, and $a=\left(a_{n}\right)$. Clearly, $0<a_{m} \leq a_{m+1}$ for $m \in \mathbb{N}$, and $\lim _{m} a_{m}=\infty$.

Assume that the space $A_{\infty}(a)$ is isomorphic to a quotient of $X$. Then there exists a linear continuous and open mapping $T$ from $X$ onto $A_{\infty}(a)$. Thus for some $k, s \in \mathbb{N}$ we have

$$
V_{1} \supset T\left(U_{k}\right) \supset T\left(U_{k+1}\right) \supset V_{s},
$$

where $V_{i}=\left\{\alpha=\left(\alpha_{n}\right) \in A_{\infty}(a): \max _{n}\left|\alpha_{n}\right| i^{a_{n}} \leq 1\right\}, i \in \mathbb{N}$.

Using Remark 8, we get

$$
d_{m}\left(U_{k+1}, U_{k}\right) \geq d_{m}\left(T\left(U_{k+1}\right), T\left(U_{k}\right)\right) \geq d_{m}\left(V_{s}, V_{1}\right), m \in \mathbb{N} .
$$

Let $n \in \mathbb{N}$ with $a_{v_{n}} \geq \max \{k, s\}$. Put $m=v_{n}$; then $a_{n}=n \geq \max \{k, s\}$. By Lemma 9 we have

$$
d_{m}\left(V_{s}, V_{1}\right) \geq|\beta| s^{-a_{m}} \geq|\beta| n^{-n}>d_{m}\left(U_{k+1}, U_{k}\right) ;
$$

a contradiction.

Similarly to the proof of Theorem 10 one can show the following

Remark 11 For any nuclear Köthe space $K(A)$ with $A=\left(a_{n, k}\right)$ there exists a nondecreasing sequence $\left(t_{n}\right) \subset \mathbb{N}$ with $\lim _{n} t_{n}=\infty$ such that for $B=\left(b_{n, k}\right)$ with $b_{n, k}=$ $a_{t_{n}, k}, n, k \in \mathbb{N}$, the nuclear Köthe space $K(B)$ is not isomorphic to a quotient of $K(A)$.

By Theorems 7 and 10, we obtain

Corollary 12 There is no nuclear Fréchet space X such that any nuclear Köthe space is isomorphic to a quotient of $X$.

\section{References}

[1] N. De Grande-De Kimpe, Non-archimedean Fréchet spaces generalizing spaces of analytic functions. Nederl. Akad. Wetensch. Indag. Mathem. 44(1982), 423-439.

[2] N. De Grande-De Kimpe, J. Kạkol, C. Perez-Garcia and W. H. Schikhof, Orthogonal sequences in non-archimedean locally convex spaces. Indag. Mathem. N.S. 11(2000), 187-195.

[3] $\longrightarrow$ Orthogonal and Schauder bases in non-archimedean locally convex spaces. In: $p$-adic Functional canalysis, Lecture Notes in Pure and Appl. Math. 222, Dekker, New York, 2001, 103-126.

[4] J. B. Prolla, Topics in Functional Analysis over Valued Division Rings. North-Holland Math. Studies 77, North-Holland, Amsterdam, 1982.

[5] A. C. M. van Rooij, Non-Archimedean functional analysis. Monographs and Textbooks in Pure and Applied Math. 51, Marcel Dekker, New York, 1978.

[6] W. H. Schikhof, Locally convex spaces over non-spherically complete valued fields. I-II. Bull. Soc. Math. Belg. 38(1986), 187-207, 208-224.

[7] W. H. Schikhof, Minimal-Hausdorff p-adic locally convex spaces. Ann. Math. Blaise Pascal, 2(1995), 259-266.

[8] W. Śliwa, Examples of non-Archimedean nuclear Fréchet spaces without a Schauder basis. Indag. Math. 11(2000), 607-616.

[9] Closed subspaces without Schauder bases in non-archimedean Fréchet spaces. Indag. Math. 12(2001), 261-271.

[10] $\longrightarrow$ On closed subspaces with Schauder bases in non-Archimedean Fréchet spaces. Indag. Math. 12(2001), 519-531. 
[11] On the quasi-equivalence of orthogonal bases in non-Archimedean metrizable locally convex spaces. Bull. Belg. Math. Soc. Simon Stevin 9(2002), 465-472.

[12] On universal Schauder bases in non-archimedean Fréchet spaces. Canad. Math. Bull. 47(2004), 108-118.

Faculty of Mathematics and Computer Science

A. Mickiewicz University

ul. Umultowska 87

61-614 Poznań

Poland

e-mail: sliwa@amu.edu.pl 